\title{
Produtividade e qualidade de frutos de cultivares de pepino para conser- va tipo "cornichon" no Vale do São Francisco
}

\author{
Geraldo M. de Resende; Nivaldo Duarte Costa; José Egidio Flori \\ Embrapa Semi-Árido, C. Postal 23, 56.300-000 Petrolina-PE; E-mail: gmilanez@ufla.br
}

\section{RESUMO}

Identificou-se as cultivares mais produtivas de pepino para conserva em um experimento, de setembro a novembro de 1996, no Campo Experimental de Bebedouro/Petrolina (PE). O delineamento experimental utilizado foi de casualizados completos, com 22 cultivares (Calypso, Eureka, Flurry, Francipak, Ginga AG-77, HE-601, HE-657, HE-671, HE-713, Imperial, Nautillus, Navigator, Panorama, Pioneiro, Premier, Prêmio, Primepak, SMR 18 Wisconsin, Supremo, Vlaspik, Vlasset e Vlasstar) e três repetições. A parcela experimental foi composta de quatro linhas de 3,0 m de comprimento, com espaçamento de $1,0 \times 0,30 \mathrm{~m}$. As cultivares Vlaspik (11,5 t/ha), Eureka (11,2 t/ha), Calypso (11,1 t/ha), Ginga AG-77 (10,7 t/ha), Imperial (10,6 t/ha), Prêmio (10,6 t/ha), Panorama (10,3 t/ha) HE671 (10,2 t/ha), Vlasset (10,1 t/ha), Francipak (10,0 t/ha), Supremo (9,7 t/ha), Primepak (9,5 t/ha), Navigator (9,5 t/ha) e Pioneiro (9,3 t/ ha), destacaram-se com produtividades mais elevadas, não diferindo estatisticamente entre si. O pior desempenho foi da cultivar SMR 18 Wisconsin, com produtividade comercial de frutos de $6,1 \mathrm{t} / \mathrm{ha}$. No que se refere a número de frutos por planta, observou-se para as cultivares Vlaspik, Eureka, Calypso, Ginga AG-77, Imperial, Prêmio, Panorama, HE-671, Vlasset e Francipak, valores oscilando de 22,7 a 25,7 frutos/planta, não diferindo estatisticamente entre si. Para classificação de frutos comerciais, verificou-se uma maior proporção de frutos tipo 1 (frutos com $<13,5 \mathrm{~mm}$ de diâmetro, $\pm 4,0 \mathrm{~cm}$ de comprimento) e Tipo 2 (frutos com 13,5 a 15,0 mm de diâmetro, \pm 4,0 a $4,5 \mathrm{~cm}$ de comprimento), correspondendo a um valor superior a $50 \%$ da produtividade comercial. A porcentagem de frutos não comerciais variou de 9,3 a $16,2 \%$ entre as cultivares.

Palavras-chave: Cucumis sativus, peso médio de fruto, número de frutos por planta, classsificação, rendimento.

\begin{abstract}
Yield and quality of pickling cucumber "cornichon" in the Vale do São Francisco, Brazil

In 1996, a field experiment was carried out in Petrolina, Pernambuco State, Brazil, to evaluate the yield of pickling cucumber cultivars. The experimental design was a randomized complete block with twenty-two treatments (cv Calypso, Eureka, Flurry, Francipak, Ginga AG-77, HE-601, HE-657, HE-671, HE-713, Imperial, Nautillus, Navigator, Panorama, Pioneiro, Premier, Prêmio, Primepak, SMR 18 Wisconsin, Supremo, Vlaspik, Vlasset and Vlasstar), and three replications. Experimental plots consisted of four rows, each one $3 \mathrm{~m}$ long spaced in $1.00 \times 0.30 \mathrm{~m}$. Cultivars Vlaspik (11.5 t/ha), Eureka (11.2 t/ha), Calypso (11.1 t/ha), Ginga AG-77 (10.7 t/ha), Imperial (10.6 t/ha), Prêmio (10.6 t/ha), Panorama (10.3 t/ha) HE-671 (10.2 t/ha), Vlasset (10.1 t/ha) and Francipak (10,0 t/ ha) presented the highest yield, without differences among them, while cv. SMR 18 Wisconsin presented the lowest yield (6.1 t/ha). The cvs. Vlaspik, Eureka, Calypso, Ginga AG-77, Imperial, Prêmio, Panorama, HE-671, Vlasset and Francipak presented the highest number of fruits per plant (from 22.7 to 25.7 fruits/plant), with no differences among them. Over $50 \%$ of total yield from all cvs. was considered as marketable and classified as type 1 (diameter $<13.5$ $\mathrm{mm}$ ) and type 2 (diameter $>13.5 \mathrm{~mm}$ and $<15.0 \mathrm{~mm}$ ). The percentage of non-marketable fruits varied from 9,3 to $16,2 \%$ among cultivars.
\end{abstract}

Keywords: Cucumis sativus, fruit average weight, number of fruits per plant, grading, yield.

\section{(Recebido para publicação em 23 de março de 2001 e aceito em 11 de junho de 2002)}

\begin{abstract}
A cultura do pepino para processamento ocupa posição de destaque no sul do Brasil, tendo o estado de Santa Catarina como principal produtor nacional, com uma produtividade média de 10 t/ha (EMPASC, 1988; Silva et al., 1992).
\end{abstract}

Alguns autores têm estudado a cultura do pepino para conserva. Pereira $e t$ al. (1976) verificaram produções de 2,2 a $4,3 \mathrm{t} / \mathrm{ha}$ de frutos comerciais $(6 \mathrm{a} 9 \mathrm{~cm}$ de comprimento), destacando-se a cultivar Cornichon de Paris com maior produção (4,3 t/ha) e número de frutos por planta (2,3 frutos). Santos et al. (1979), comparando híbridos e cultivares de pepino conduzidos no sistema rasteiro, observaram maiores produções para os híbridos, tendo os híbridos Conda (26,5 t/ha), Pionner (23,4 t/ha) e Explorer $(22,8 \mathrm{t} / \mathrm{ha})$ se destacado na produção de frutos comerciais (7 a $10 \mathrm{~cm}$ de comprimento). Silva et al. (1988; 1992), obtiveram os maiores rendimentos, em plantio rasteiro, em setembro, onde observaram uma produtividade média de $23,8 \mathrm{t} / \mathrm{ha}$ de frutos comerciais ( 6 a 12 cm de comprimento) com $357 \mathrm{~g} /$ planta, peso médio do fruto de $33,0 \mathrm{~g}$ e 10,8 frutos/planta, na densidade de 66.666 plantas/ha, utilizando as cultivares Score e Ginga AG-77.
Utilizando a classificação "cornichon" (frutos comerciais variando de 4,0 a 5,5 cm de comprimento), Resende \& Pessoa (1996), encontraram produtividades variando de 3,1 a 10,2 $\mathrm{t} /$ ha, destacando-se as cultivares Tamor, Indaial, Colônia, Score, Ginga AG-77, Levina e Primepak com produtividades acima de $8,8 \mathrm{t} / \mathrm{ha}$, sendo que o peso médio de fruto e número de frutos por planta variaram entre 5,6 a 6,1 g/fruto e 8,3 a 27,8 frutos por planta, respectivamente. Com relação à percentagem de frutos não comerciais observou-se uma variação de 11,4 a 16,1\%. Para classificação de frutos comerciais, verificaram 
para as cultivares avaliadas valores superiores a $60 \%$ de frutos tipo 1 e 2 (frutos com $\pm 4,0$ a 4,5 cm), de maior valor comercial, à exceção da cultivar Pérola que apresentou 56,1\%. Com relação a frutos comerciais tipos 3 constatou uma oscilação de 20,8 a $28,6 \%$ e tipo 4 de 12,0 a $15,3 \%$ (frutos com $\pm 4,5$ a $5,0 \mathrm{~cm}$ e $\pm 5,0$ a $5,5 \mathrm{~cm}$, respectivamente).

No Nordeste do Brasil há uma grande deficiência de opções de olerícolas para cultivo em áreas irrigadas que possam ser processadas industrialmente, principalmente culturas que possam utilizar a infra-estrutura agroindustrial já instalada na região. De acordo com Rabelo et al. (1990), o desenvolvimento agroindustrial é um meio para aumentar a eficiência da produção na agricultura. Segundo estes autores, existe uma grande deficiência na produção e qualidade das matérias primas nos pólos de irrigação, salientando que a atividade é direcionada para um pequeno número de culturas industriais (tomate e aspargo), obrigando as agroindústrias a operarem com ociosidade ou a importarem matéria prima de outras regiões. Assim a identificação de novas opções de olerícolas que possam ser processadas industrialmente é uma das prioridades para as áreas irrigadas do Nordeste como forma de consolidar as agroindústrias já instaladas, como também de viabilizar a implantação de novas unidades. Neste contexto o presente trabalho objetivou identificar cultivares de pepino para conserva ("cornichon") mais produtivas e com melhor qualidade de frutos para as condições do Vale do São Francisco, como uma alternativa agroindustrial para a região.

\section{MATERIAL E MÉTODOS}

O experimento foi conduzido no Campo Experimental de Bebedouro da Embrapa Semi-Árido em Petrolina (PE), cujas coordenadas geográficas são $9^{\circ} \mathrm{e}$ $9^{\prime}$ de latitude Sul e $40^{\circ}$ e $29^{\prime}$ de longitude Oeste e altitude de 365,5 m (Amorim Neto, 1989).

O delineamento experimental utilizado foi de blocos casualizados completos, com 22 tratamentos (Calypso, Eureka, Flurry, Francipak, Ginga AG77, HE-601, HE-657, HE-671, HE-713,
Imperial, Nautillus, Navigator, Panorama, Pioneiro, Premier, Prêmio, Primepak, SMR 18 Wisconsin, Supremo, Vlaspik, Vlasset e Vlasstar) e três repetições. À exceção da cultivar SMR 18 Wisconsin de polinização aberta todas as demais são híbridos. As parcelas experimentais constaram de quatro linhas de 3,0 m, com espaçamento de 1,0 x $0,30 \mathrm{~m}$, plantas, com duas plantas por cova, sendo que as linhas centrais constituíram a área útil, sendo a semeadura feita em 12 de setembro de 1996.

A adubação de plantio foi de $150 \mathrm{~kg} /$ ha de sulfato de amônio, $220 \mathrm{~kg} / \mathrm{ha}$ de superfosfato simples e $65 \mathrm{~kg} /$ ha de cloreto de potássio, sendo realizadas duas coberturas com $150 \mathrm{~kg} / \mathrm{ha}$ de sulfato de amônio, fazendo-se a primeira após o desbaste e a segunda 15 dias após a primeira.

Foram realizadas irrigações duas vezes por semana, sendo a cultura mantida no limpo através de capinas manuais e conduzida sob o sistema rasteiro. Os tratos fitossanitários empregados foram os usualmente utilizados em pepino, realizados somente até o início da floração (30 dias após a semeadura). Após este período, foram utilizados somente produtos à base de enxofre e detergente neutro, pulverizados semanalmente até o fim da colheita.

As colheitas foram feitas diariamente, iniciando-se aos 36 dias após a semeadura e estendendo-se por um período de 40 dias. Foram avaliadas a produtividade comercial e não comercial $(\mathrm{t} /$ ha); peso médio de fruto ( $\mathrm{g}$ ) e o número de frutos por planta. Os frutos foram classificados em comerciais (Tipo 1: $<13,5 \mathrm{~mm}$ de diâmetro, $\pm 4,0 \mathrm{~cm}$ de comprimento; Tipo 2: 13,5 a 15,0 mm de diâmetro, $\pm 4,0$ a $4,5 \mathrm{~cm}$ de comprimento; Tipo 3: 15,1 a 17,0 mm de diâmetro, $\pm 4,5$ a $5,0 \mathrm{~cm}$ de comprimento; Tipo 4: 17,1 a 19,0 mm de diâmetro, \pm 5,0 a $5,5 \mathrm{~cm}$ de comprimento) e não comerciais (frutos com mais de 19,0 mm de diâmetro, deformados e afilados) de acordo com Resende \& Pessoa (1996). Os dados foram submetidos à análise de variância, sendo as médias comparadas pelo teste de Scott-Knott ao nível de 5\% de probabilidade. Os dados originais de porcentagem foram transformados para arco-seno $\sqrt{P / 100}$ para efeito de análise estatística.

\section{RESULTADOS E DISCUSSÃO}

As produtividades comerciais variaram de 6,1 a $11,5 \mathrm{t} /$ ha nas diferentes cultivares (Tabela 1). Os melhores desempenhos foram obtidos pelas cultivares Vlaspik (11,5 t/ha), Eureka (11,2 t/ ha), Calypso (11,1 t/ha), Ginga AG-77 (10,7 t/ha), Imperial (10,6 t/ha), Prêmio (10,6 t/ha), Panorama (10,3 t/ha) HE$671(10,2 \mathrm{t} / \mathrm{ha})$, Vlasset $(10,1 \mathrm{t} / \mathrm{ha})$, Francipak (10,0 t/ha), Supremo (9,7 t/ ha), Primepak (9,5 t/ha), Navigator $(9,5$ $\mathrm{t} / \mathrm{ha}$ ) e ioneiro (9,3 $\mathrm{t} / \mathrm{ha})$, que não diferiram estatisticamente entre si. O pior desempenho foi da cultivar SMR 18 Wisconsin, com produtividade comercial de frutos de 6,1 t/ha. Estes resultados são superiores aos observados por Pereira et al. (1976) que verificaram produções variando de 2,2 a 4,3 t/ha de frutos comerciais (6 a $9 \mathrm{~cm}$ de comprimento) e similares à produção média de Santa Catarina de 10 t/ha (EMPASC, 1988; Silva et al. 1992), salientando que esta produção baseia-se em frutos comerciais de 6 a $12 \mathrm{~cm}$ de comprimento, enquanto a classificação de frutos comerciais adotado no presente trabalho foi de 4,0 a $5,5 \mathrm{~cm}$ de comprimento. Assim como são semelhantes aos resultados obtidos por Resende \& Pessoa (1996), também utilizando a classificação "cornichon".

Para o peso médio de frutos não se observou diferenças significativas (Tabela 1 ), verificando-se valores entre 6,1 e $6,9 \mathrm{~g} /$ fruto entre as cultivares. A pequena variação percentual entre os pesos médios de frutos das cultivares evidencia um bom padrão (tamanho do fruto) destas, como ideais para processamento. Resende \& Pessoa (1996) encontraram pesos médios similares aos obtidos no presente trabalho com variações de 5,6 a 6,1 g/fruto. Ao se comparar número de frutos por planta (Tabela 1), observa-se que as cultivares Vlaspik, Eureka, Calypso, Ginga AG-77, Imperial, Prêmio, Panorama, HE-671, Vlasset e Francipak, destacaram-se com valores oscilando de 22,7 a 25,7 frutos/planta, não diferindo estatisticamente entre si, sendo a pior performance da cultivar SMR 18 Wisconsin (14,1 frutos/planta). Pereira 
Tabela 1. Produtividade comercial, peso médio de frutos e número de frutos por planta de cultivares de pepino para indústria. Petrolina, Embrapa Semi-Árido, 1996*.

\begin{tabular}{|c|c|c|c|c|}
\hline Cultivares & $\begin{array}{c}\text { Produtividade comercial } \\
\text { (t/ha) }\end{array}$ & $\begin{array}{l}\text { Peso médio de frutos } \\
\text { (g) }\end{array}$ & \multicolumn{2}{|c|}{ Número de frutos/planta } \\
\hline Vlaspik & $11,46 a$ & $6,63 \mathrm{a}$ & $25,67 \mathrm{a}$ & \\
\hline Eureka & 11,20 a & $6,80 \mathrm{a}$ & $24,54 \mathrm{a}$ & \\
\hline Calypso & $11,10 \mathrm{a}$ & 6,73 a & $24,77 \mathrm{a}$ & \\
\hline Ginga AG-77 & $10,73 \mathrm{a}$ & $6,63 \mathrm{a}$ & $24,30 \mathrm{a}$ & \\
\hline Imperial & $10,63 a$ & $6,83 \mathrm{a}$ & $23,43 \mathrm{a}$ & \\
\hline Prêmio & $10,57 \mathrm{a}$ & 6,83 a & $23,20 \mathrm{a}$ & \\
\hline Panorama & $10,28 a$ & $6,70 \mathrm{a}$ & $22,93 \mathrm{a}$ & \\
\hline HE-671 & $10,23 a$ & $6,67 \mathrm{a}$ & $22,97 \mathrm{a}$ & \\
\hline Vlasset & $10,13 a$ & $6,57 \mathrm{a}$ & $23,03 \mathrm{a}$ & \\
\hline Francipak & $10,00 \mathrm{a}$ & $6,63 \mathrm{a}$ & $22,67 \mathrm{a}$ & \\
\hline Supremo & 9,70 a & $6,90 \mathrm{a}$ & 21,06 & \\
\hline Primepak & 9,53 a & $6,63 \mathrm{a}$ & 21,60 & $\mathrm{~b}$ \\
\hline Navigator & 9,46 a & $6,50 \mathrm{a}$ & 21,80 & $\mathrm{~b}$ \\
\hline Pioneiro & $9,30 \mathrm{a}$ & $6,70 \mathrm{a}$ & 20,90 & $\mathrm{~b}$ \\
\hline Vlasstar & $8,87 \quad b$ & $6,53 \mathrm{a}$ & 20,47 & $\mathrm{~b}$ \\
\hline Flurry & $8,20 \mathrm{~b}$ & $6,63 \mathrm{a}$ & 18,47 & c \\
\hline Nautillus & $8,13 \mathrm{~b}$ & $6,50 \mathrm{a}$ & 18,73 & c \\
\hline HE-657 & $8,10 \quad b$ & $6,50 \mathrm{a}$ & 18,73 & c \\
\hline HE-713 & $8,08 \quad b$ & $6,57 \mathrm{a}$ & 18,46 & c \\
\hline HE-601 & $7,56 \quad b$ & $6,13 a$ & 18,50 & c \\
\hline Premier & $7,53 \mathrm{~b}$ & $6,53 a$ & 17,33 & c \\
\hline SMR 18 Wisconsin & $6,10 \quad c$ & $6,50 \mathrm{a}$ & 14,07 & d \\
\hline C.V. (\%) & 9,45 & 3,09 & 8,42 & \\
\hline
\end{tabular}

*Médias seguidas de mesma letra nas colunas não diferem entre si, pelo teste Scott-Knott, a 5\% de probabilidade.

et al. (1976) encontraram resultados inferiores para número de frutos por planta (2,3 frutos/planta) para a cultivar Cornichon de Paris, assim como Silva et al. (1988), que verificaram média de 10,8 frutos por planta para as cultivares Score e Ginga AG-77, na mesma densidade de plantas do presente trabalho e frutos de maior comprimento ( 6 a $12 \mathrm{~cm}$ ). Resultados similares foram relatados por Resende \& Pessoa (1996), que verificaram para as melhores cultivares uma variação de 25,2 a 27,8 frutos /planta.

Para classificação de frutos comerciais (Tabela 2), verifica-se uma maior proporção de frutos tipos 1 e 2, de maior valor comercial, tendo todas as cultivares alcançado valores superiores a $50 \%$. Para frutos tipo 1 e 2 verificou-se variações de 12,0 a $42,5 \%$, sendo que para frutos comerciais tipos 3 e 4 , de menores cotações no mercado, verificou-se pequenas variações 18,9 a $27,0 \%$, demonstrando uma ótima qualidade de frutos para processamento com pequena variação no comportamento das cultivares. Resende \& Pessoa (1996) verificaram para as cultivares avaliadas, valores superiores a $60 \%$ de frutos tipo 1 e 2 de maior valor comercial, à exceção da cultivar Pérola que obteve 56,1\%. Para frutos tipo 3 e 4 observaram variações de 12,0 a $28,6 \%$.

A porcentagem de frutos não comerciais oscilou de 9,3 a $16,2 \%$ entre as cultivares (Tabela 2), sobressaindo-se as cultivares Nautillus, Panorama, HE-671, SMR 18 Wisconsin e Imperial com as maiores porcentagens (acima de 14\%). Resende \& Pessoa (1996), encontraram resultados semelhantes com variações de 11,4 a $16,1 \%$, enquanto Silva et al. (1988) verificaram para as cultivares
Score e Ginga AG-77, 28,2\% de frutos não comerciais na mesma densidade populacional, no entanto, classificando os frutos comerciais de 6 a $12 \mathrm{~cm}$ de comprimento, como também Cerne (1994) encontrou até 30\% para a cultivar Levina, resultados bem superiores aos apresentados no presente trabalho.

Com relação a ciclo vegetativo verificou-se uma variação de 73 a 75 dias para as cultivares avaliadas, pequenas diferenças de ciclo, que não chegam a viabilizar um provável escalonamento da produção. Para precocidade e cor do fruto, observou-se para as cultivares o mesmo período de colheita inicial, à exceção da cultivar Francipak, que iniciou a sua colheita sete dias após as demais cultivares. No que se refere à ocorrência de pragas e doenças, observouse na última semana de colheita a ocorrência de mosca-branca (Bemisia sp.) 
Tabela 2. Classificação de frutos comerciais segundo o diâmetro e comprimento e não comerciais, em percentagem de cultivares de pepino para indústria. Petrolina, Embrapa Semi-Árido, 1996*.

\begin{tabular}{|c|c|c|c|c|c|c|c|}
\hline \multirow{2}{*}{ Cultivares } & \multicolumn{6}{|c|}{ Classificação de frutos comerciais } & \multirow{2}{*}{$\begin{array}{c}\text { Frutos não } \\
\text { comerciais (\%) }\end{array}$} \\
\hline & \multicolumn{2}{|l|}{ Tipo 1} & \multicolumn{2}{|c|}{ Tipo 2} & Tipo 3 & Tipo 4 & \\
\hline Vlaspik & $18,58 \mathrm{a}$ & & 37,33 & $\mathrm{~d}$ & $24,08 \quad b$ & $20,01 b$ & $12,12 b$ \\
\hline Eureka & 13,70 & $\mathrm{c}$ & 38,50 & c & 26,58 a & $21,22 b$ & $13,62 \mathrm{a}$ \\
\hline Calypso & 15,00 & $\mathrm{c}$ & 36,13 & $d$ & 25,84 a & $23,03 a$ & $13,54 \mathrm{a}$ \\
\hline Ginga AG-77 & 15,37 & $\mathrm{c}$ & 40,47 & $b$ & $23,37 \quad c$ & $20,79 b$ & $11,37 b$ \\
\hline Imperial & 13,91 & $\mathrm{c}$ & 37,94 & c & 25,81 a & $22,34 \mathrm{a}$ & 14,31 a \\
\hline Prêmio & 12,63 & $\mathrm{c}$ & 40,94 & $\mathrm{~b}$ & $24,19 \quad b$ & $22,24 \mathrm{a}$ & $13,84 \mathrm{a}$ \\
\hline Panorama & 13,68 & $\mathrm{c}$ & 37,83 & c & 25,44 a & $23,05 \mathrm{a}$ & $16,12 \mathrm{a}$ \\
\hline HE-671 & 14,64 & $\mathrm{c}$ & 39,76 & $\mathrm{~b}$ & $23,14 \quad c$ & $22,46 \mathrm{a}$ & 15,59 a \\
\hline Vlasset & 14,53 & c & 41,20 & & $24,88 \quad b$ & $19,39 \mathrm{~b}$ & $10,88 \mathrm{~b}$ \\
\hline Francipak & 13,14 & c & 42,39 & & $24,47 \quad b$ & $20,00 \mathrm{~b}$ & $10,58 \mathrm{~b}$ \\
\hline Supremo & 13,11 & c & 40,66 & $\mathrm{~b}$ & 26,12 a & $20,11 b$ & $10,10 \mathrm{~b}$ \\
\hline Primepak & 17,13 & b & 39,20 & c & $23,00 \quad c$ & $20,67 b$ & $13,47 \mathrm{a}$ \\
\hline Navigator & $19,95 \mathrm{a}$ & & 38,60 & c & 21,57 & $19,88 \mathrm{~b}$ & $11,10 \mathrm{~b}$ \\
\hline Pioneiro & 14,88 & c & 39,68 & $\mathrm{~b}$ & 24,88 b & $20,56 \mathrm{~b}$ & 13,85 a \\
\hline Vlasstar & $17,91 \mathrm{a}$ & & 40,72 & $\mathrm{~b}$ & 21,93 & $19,44 \mathrm{~b}$ & $11,19 \mathrm{~b}$ \\
\hline Flurry & 13,84 & $\mathrm{C}$ & 36,38 & $d$ & $24,85 \quad b$ & $24,93 \mathrm{a}$ & $10,80 \mathrm{~b}$ \\
\hline Nautillus & $17,96 \mathrm{a}$ & & 42,53 & & $20,57 \quad d$ & $18,94 \mathrm{~b}$ & $16,22 \mathrm{a}$ \\
\hline HE-657 & 11,99 & $\mathrm{C}$ & 38,31 & c & 26,99 a & $22,71 \mathrm{a}$ & $12,47 b$ \\
\hline HE-713 & 13,76 & $\mathrm{c}$ & 39,33 & c & $24,07 \quad b$ & $22,84 \mathrm{a}$ & $9,26 \mathrm{~b}$ \\
\hline HE-601 & 15,99 & b & 41,60 & & $22,76 \quad c$ & $19,65 \mathrm{~b}$ & $12,74 b$ \\
\hline Premier & 16,06 & b & 38,62 & c & $25,55 \mathrm{a}$ & $19,77 b$ & $12,76 \mathrm{~b}$ \\
\hline SMR 18 Wisconsin & 13,68 & $\mathrm{c}$ & 39,69 & $\mathrm{~b}$ & 25,85 a & $21,23 b$ & $15,22 \mathrm{a}$ \\
\hline C.V. (\%) & 4,87 & & 2,18 & & 2,98 & 4,84 & 10,13 \\
\hline
\end{tabular}

*Médias seguidas de mesma letra nas colunas não diferem não diferem entre si, pelo teste Scott-Knott, a 5\% de probabilidade.

que aparentemente, por já estar no fim do ciclo vegetativo, não trouxe grandes prejuízos para a cultura. Não verificou-se a ocorrência de doença no experimento.

Apesar de não se destacar em termos de produtividade, a cultivar HE-713 pela menor percentagem de frutos não comerciais $(9,3 \%)$, é uma boa opção para compor futuros trabalhos de melhoramento, visando uma melhor qualidade de fruto. O bom desempenho das cultivares cultivares Vlaspik, Eureka, Calypso, Ginga AG-77, Imperial, Prêmio, Vlasset e Francipak, destacandose nas diferentes características analisadas, as viabiliza como uma nova alternativa agroindustrial de cultivo de pepino para processamento tipo "cornichon", para as condições do Vale do São Francisco.

\section{LITERATURA CITADA}

AMORIM NETO, M.S. Informações meteorológicas dos Campos Experimentais de Bebedouro e Mandacaru, Petrolina,PE. Petrolina: EMBRAPA-CPATSA, 1989. 58 p. (EMBRAPACPATSA, Documentos, 57).

CERNE, M. Different agrotextiles for direct covering of pickling cucumbers. Acta Horticulturae, v. 371, p. 244-252, 1994.

EMPASC (Florianópolis, SC). Normas técnicas da cultura do pepino para conserva: região do Vale do ltajaí e Litoral Norte Catarinense. Florianópolis: EMPASC/ACARESC, 1988. 18 p. (Sistemas de Produção II).

PEREIRA, A.C.; KIMURA, O.; BATISTA, L.B. Avaliação de cultivares de pepino (Cucumis sativus L.) para processamento. Revista de Olericultura, Lavras, v. 16, p. 147-148, 1976.

RABELO, J.L.C.; COELHO, J.P.; SANTOS, J.A.N. Estudos sobre a agroindústria no Nordeste: situação atual a perspectiva da produção irrigada. Fortaleza; BNB/ETENE, v. 2, 1990. 139 p.
RESENDE, G.M.; PESSOA; H.B.S.V. Produção de pepino para indústria no Perímetro Irrigado do Gorutuba. Horticultura Brasileira, Brasília, v. 14, n. 2, p. 220-222, 1996.

SANTOS, A.M.; MAGALHAES, A.E.; MORAES, E.C.; OLIVEIRA, J.J. Competição entre cultivares e híbridos de pepino para indústria, ln: CONGRESSO BRASILEIRO DE OLERICULTURA, 17, 1977, Juazeiro. Anais... Petrolina-PE: EMBRAPA-CPATSA, 1979. p. 130131.

SILVA, A.C.F.; AGOSTINI, I.; MULLER, J.J.V.; VIZZOTO, V.J. Efeito de densidades populacionais sobre a produtividade de pepino para conserva. Horticultura Brasileira, Brasília, v. 10, n. 1, p. 28-29, 1992.

SILVA, A.C.F.; MULLER, J.J.V.; DALL'AGNOL, I.; AGOSTINI, I. Épocas de semeadura e densidade populacional de pepino para conserva. Florianópolis: EMPASC, 1988. 16 p. (Comunicado Técnico 115). 